\title{
Novel Flow Control for Fully Adaptive Routing in Cache-coherent NoCs
}

\author{
Sheng Ma, Zhiying Wang, Member, IEEE, Natalie Enright Jerger, Senior Member, IEEE, \\ Li Shen and Nong Xiao, Member, IEEE
}

\section{Supplementary file}

\begin{abstract}
This paper contains the supplementary material to the paper submitted to IEEE TPDS entitled "Novel Flow Control for Fully Adaptive Routing in Cache-coherent NoCs" (labeled as "main file" in this paper). In Sec. 1, we prove that if a routing algorithm with WPF is deadlock-free, then this algorithm with conservative VC re-allocation is also deadlock-free. In Sec. 2, we provide further insights for the performance trends of routing algorithms by analyzing the buffer utilization. In Sec. 3, we present the full system performance analysis. In Sec. 4, we show the recursive algorithm to calculate the allowable escape VC. In Sec. 5, we perform sensitivity study on several configuration parameters. In Sec. 6, we provide further discussion about the designs.
\end{abstract}

\section{Logical Equivalence of Alg AND $A l g+W P F$}

Sec. 4.1 proposes Theorem 1, which declares that if a fully adaptive routing algorithm with conservative VC re-allocation (named as $A l g$ ) is deadlock-free, then applying WPF on this routing algorithm (named as $A l g+W P F)$ is also deadlock-free. This section proves that if $A l g+W P F$ is deadlock-free, then $A l g$ is deadlock-free as well. In addition to Assumption 1 in Sec. 4.1 of the main file, this proof needs another simple assumption about the routing algorithm.

Assumption 2. The definition of a routing algorithm is independent of the packet length.

Assumption 2 implies that if two packets are injected from the same source and destined to the same node, they can use the same set of VCs even though these packets have different lengths. This assumption is generally used in many previous theories [2], [3], [6], [7], [8], [9], [10], [12], [18], [21], [24], [25]. We prove the following theorem.

Theorem 3: If $A l g+W P F$ is deadlock-free, then $A l g$ is also deadlock-free.

Description: This proof is similar to the proof of Theorem 1 in the main file. We prove that if $A l g$ has a deadlock configuration, then $A l g+W P F$ has a deadlock configuration as well. Use Config in $_{\text {in }} 1$ as an example. We replace packet $P_{i}$ with packet $P_{i}^{\prime}$ to completely fill all VCs. The source and destination of $P_{i}^{\prime}$ are the same as $P_{i}$, while $P_{i}^{\prime}$ may have more flits than $P_{i}$ to completely fill the VCs. We prove

- Sheng Ma, Zhiying Wang, Li Shen and Nong Xiao are with the State Key Laboratory of High Performance Computing, National University of Defense Technology, China. Sheng Ma is also with the National Key Laboratory for Paralleling and Distributed Processing, NUDT. E-mail: \{masheng, zywang, lishen, nongxiao\}@nudt.edu.cn

This research was mostly done when Sheng Ma was a visiting international student at the University of Toronto.

- Natalie Enright Jerger is with the Department of Electrical and Computer Engineering, University of Toronto, Toronto, Ontario, Canada. E-mail: enright@eecg.toronto.edu

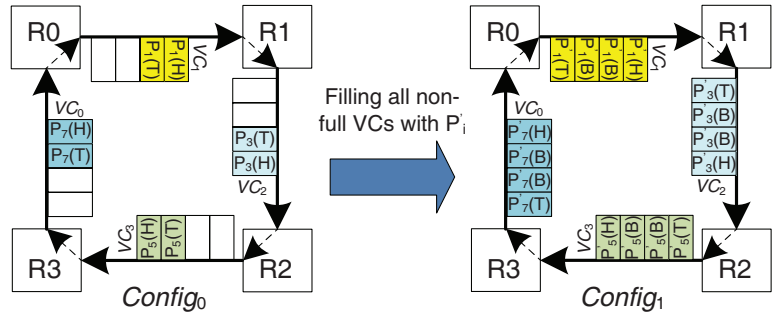

Fig. 1. The construction of a new configuration.

that $A l g+W P F$ can achieve Config, and Config is a deadlock configuration. However, $A l g+W P F$ is deadlock-free, thus there is no such configuration.

Proof: By contradiction. If $\mathrm{Alg}$ is not deadlock-free, then there is a deadlock configuration $\left(\mathrm{Config}_{0}\right)$ in which a set of packets, $P_{\text {set }_{0}}$, are waiting on VCs held by other packets in $P_{\text {set }}$. We prove that there is a deadlock configuration for $A l g+W P F$ in three steps.

Step 1: We build a new configuration based on Config $g_{0}$. Consider each packet $P_{i}$ in $P_{\text {set }}$. If $P_{i}$ does not completely fill all VCs where its flits reside, we replace $P_{i}$ with packet $P_{i}^{\prime}$ to completely fill all VCs occupied by $P_{i}$. The source and destination of $P_{i}^{\prime}$ are the same as $P_{i}$, and the length of $P_{i}^{\prime}$ is larger than $P_{i}$. If $P_{i}$ completely fills all VCs where its flits reside, $P_{i}^{\prime}$ is the same as $P_{i}$. We label the new configuration as Config ${ }_{1}$, and the set of packets in Config as $_{\text {set }_{1}}$.

Step 2: We prove that when the network is routed by $A l g+W P F$, all packets in $P_{\text {set }}$ can move to their

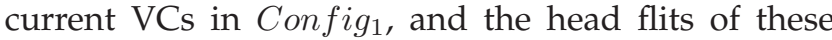
packets are at VC heads. For each packet $P_{i}^{\prime}$ in $P_{\text {set }}$, we consider its corresponding packet $P_{i}$ in Config . We further consider each hop hop $k$ of $P_{i}$ when it is routed by Alg. Assume the head flit of $P_{i}$ moves to $V C_{k}$ 's head during hop $p_{k}$. The routing algorithm allows $P_{i}$ to use $V C_{k}$. Although $P_{i}^{\prime}$ and $P_{i}$ may have different packet lengths, they have the same source and destination information. Based on Assumption 2, the routing algorithm allows $P_{i}^{\prime}$ to use $V C_{k}$ as well.

Since $A l g+W P F$ is deadlock-free, $V C_{k}$ must become available at some time. Based on Assumption 


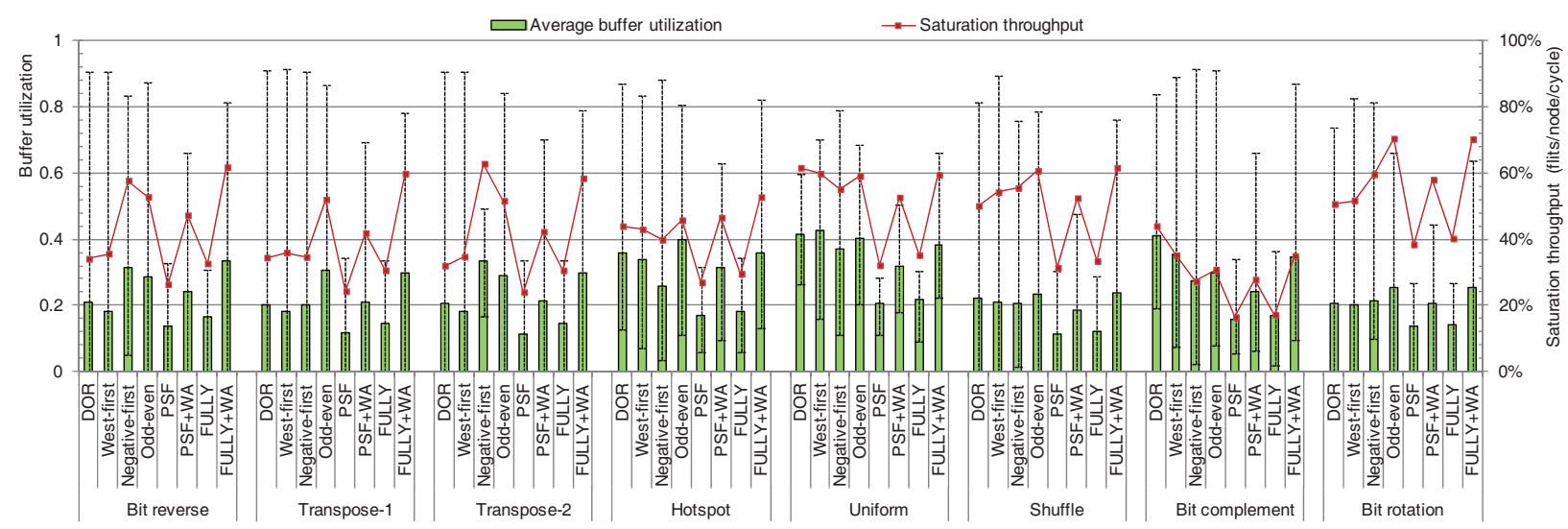

Fig. 2. The buffer utilization and throughput in saturation for several routing algorithms.

1 in the main file, $P_{i}^{\prime}$ has some possibility to request $V C_{k}$. There are two cases when $P_{i}^{\prime}$ requests $V C_{k}$.

2.1) $V C_{k}$ is empty. $V C_{k}$ can be re-allocated to $P_{i}^{\prime}$ with conservative VC re-allocation. Thus, the head flit of $P_{i}^{\prime}$ will move to $V C_{k}$ 's head.

2.2) $V C_{k}$ is not empty. But $V C_{k}$ already received the tail flit of last allocated packet and still has enough buffers for $P_{i}^{\prime}$. WPF can re-allocate $V C_{k}$ to $P_{i}^{\prime}$. Yet, when $P_{i}^{\prime}$ reaches $V C_{k}$, its head flit is not at $V C_{k}$ 's head. Considering $A l g+W P F$ is deadlock-free, the packets in $V C_{k}$ before $P_{i}^{\prime}$ must be sent out in limited time. Then, the head flit of $P_{i}^{\prime}$ is at $V C_{k}$ 's head.

Considering 2.1) and 2.2) together, if the head flit of $P_{i}$ moves to the head of a VC during any hop when routed by $A l g$, the head flit of $P_{i}^{\prime}$ can also move to the same VC head when routed by $A l g+W P F$. Thus, when routed by $A l g+W P F, P_{i}^{\prime}$ can be routed to its current VC(s) in Config $g_{1}$, and the head flit of $P_{i}^{\prime}$ is at the VC head.

Step 3: We prove that Config 1 is a deadlock configuration for $A l g+W P F$. Since all VCs in Config 1 are completely filled, all packets in $P_{\text {set }}$ still wait for VCs held by other packets in $P_{\text {set }}$, even with WPF applied. Con $\mathrm{fig}_{1}$ is a deadlock configuration for $A l g+W P F$. But $A l g+W P F$ is deadlock-free, so there is no deadlock configuration. Thus, $A l g$ is deadlockfree as well.

Theorem 1 of the main file declares that if Alg is deadlock-free, then $A l g+W P F$ is deadlock-free as well. Theorem 3 declares that if $A l g+W P F$ is deadlock-free, then $\mathrm{Alg}$ is deadlock-free as well. Considering these two theorems together, the deadlock freedom of $A l g+W P F$ is logically equivalent to the deadlock freedom of Alg. Therefore, the WPF optimization of traditional wormhole flow control does not have any influence on the deadlock freedom.

\section{Buffer Utilization of Routing Al- GORITHMS}

Sec. 5.1 of the main file measures the performance of several fully adaptive, partially adaptive and deterministic routing algorithms. This section provides further insights of the performance trends by analyzing the buffer utilization of all evaluated designs. Fig. 2 illustrates the average buffer utilization of all network VCs in saturation for eight synthetic traffic patterns. The maximum and minimum rates are given by the error bars. Fig. 2 also shows the saturation throughput supported by routing algorithms. There are several important insights.

First, buffers are used to support high throughput. The saturation throughput of routing algorithms is related to the buffer utilization. Higher average buffer utilization generally leads to higher saturation throughput. This trend is obvious in bit complement. Bit complement sends traffic from node $\left\{s_{3}, s_{2}, s_{1}, s_{0}\right\}$ to node $\left\{\neg s_{3}, \neg s_{2}, \neg s_{1}, \neg s_{0}\right\}$, which has the largest average hop count [14], and puts the highest pressure on the buffers among all evaluated traffic patterns.

Second, for the same routing algorithm in different traffic patterns, there may be fluctuations between the buffer utilization and the saturation throughput. For example, even though DOR shows much higher buffer utilization for bit complement than shuffle, its saturation throughput for bit complement is lower than shuffle. These fluctuations are due to that the same routing algorithm uses different VCs for different patterns; the network becomes saturated in different status. Similarly, since some algorithms use different VCs in the same traffic pattern, there may be slight fluctuations as well. For example, even though the average buffer utilization of west-first is slightly lower than DOR for bit reverse, its saturation throughput is higher than DOR.

Third, since all fully adaptive routing algorithms, including PSF, PSF+WA, FULLY and FULLY+WA, use the same set of VCs for the same traffic pattern, their saturation throughput is roughly proportional to the buffer utilization. For instance, in bit reverse, PSF+WA's average buffer utilization is $78.3 \%$ higher than PSF, making it perform $79.5 \%$ better than PSF. Similarly, PSF+WA has $67.4 \%$ higher average buffer utilization than PSF in shuffle, and its saturation throughput is $67.2 \%$ higher than PSF.

Fourth, the network becomes saturated when some resources become saturated [4]. For most patterns and 


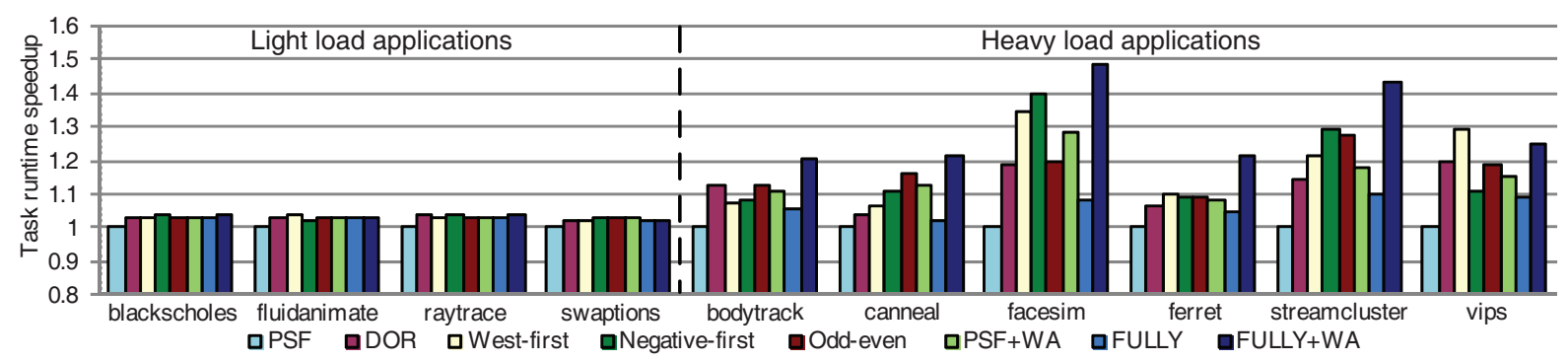

Fig. 3. System speedup for PARSEC benchmarks.

most algorithms, the bottleneck is VCs; networks become saturated when some VCs have more than $80 \%$ utilization, including DOR, west-first, negative-first and odd-even in most patterns. When the algorithm provides abundant adaptiveness, it distributes traffic more uniformly among VCs, preventing them from becoming the bottleneck. Instead, the bottleneck may be the crossbar or network interface [23]. For example, since negative-first offers adaptiveness for all traffic in tranpose-2, the network becomes saturated even when the maximum buffer utilization is only $49.2 \%$. Similarly, when in saturation, the maximum buffer utilization of PSF+WA and FULLY+WA is lower than partially adaptive routing and DOR.

Fifth, PSF and FULLY are limited by conservative VC re-allocation; their maximum buffer utilization for evaluated patterns is less than $40 \%$. Applying WPF on adaptive VCs and aggressive VC re-allocation on escape VCs greatly increases buffer utilization, which proportionally improves performance. FULLY supports higher routing flexibility than PSF, which brings higher buffer utilization and performance.

\section{Evaluation ON PARSEC WORKLOAdS}

\subsection{Methodology and Configuration}

To measure full-system performance, we leverage FeS2 [16] for x86 simulation and BookSim for NoC simulation. FeS2 is implemented as a module for Simics [15]. We run PARSEC benchmarks [1] with 16 threads on a 16-core CMP, which is organized as a $4 \times 4$ mesh. Prior research shows the frequency of simple cores in a many-core platform can be optimized to $5 \sim 10 \mathrm{GHZ}$, while the frequency of NoC router is limited by the allocator speed [5]. We assume cores are clocked $5 \times$ faster than the network. Cache lines are 64 bytes; long packets are 5 flits long with a 16-byte flit width. We use a distributed, directorybased MOESI coherence protocol which needs 4 VNs for protocol-level deadlock freedom. Each VN has 2 VCs; each VC is 4 flits deep. The router pipeline is the same as described in Sec. 4.4 of the main file. All benchmarks use the simsmall input sets. The total runtime is the performance metric. TABLE 1 gives the system configuration.

\subsection{Performance}

Fig. 3 shows the speedups relative to PSF for PARSEC workloads. We divide the 10 applications into 2 classes. For blackscholes, fluidanimate, raytrace
TABLE 1. Full system simulation configuration.

\begin{tabular}{|l|l|}
\hline$\#$ of cores & $16,4 \times 4$ Mesh \\
\hline L1 cache $(\mathrm{D} \& \mathrm{I})$ & private, 4 -way, 32KB each \\
\hline L2 cache & private, 8 -way, 512KB each \\
\hline Cache coherence & MOESI distributed directory \\
\hline
\end{tabular}

and swaptions, different algorithms perform similarly. The working sets of these applications fit into the caches and their computation phases consist of few synchronization points, leading to a lightly loaded network. The system performance of these applications is unaffected by techniques that improve the network throughput, such as sophisticated routing algorithms.

However, the routing algorithms affect the other 6 applications. These applications have heavier loads than previous 4 applications. Yet, their average aggregate loads during the running periods are lower than the network saturation points. The highest average aggregate injection rate for canneal is $12.3 \%$ flits/node/cycle, which is below the saturation points for all designs. Two factors bring performance improvements for these 6 applications. First, these applications exhibit period bursty communication and the synchronization primitives create one hotspot inside the network. The bursty communication and the hotspot make the network operate past saturation at times during the running period. Thus, these applications benefit from routing algorithms with higher throughputs. Second, short packets are critical in cache-coherent many-core platforms; they carry timecritical control messages that are often on the application's critical path. These short packets can affect the execution time significantly. The novel flow control reduces the packet latency, especially under heavy loads, which brings performance gains. For example, FULLY+WA has $48.5 \%$ and $43.0 \%$ speedups over PSF for facesim and streamcluster.

Since most of vips application's bursty communication is eastbound, west-first performs best for vips. For facesim and streamcluster, negativefirst offers higher adaptiveness than odd-even, thus achieving better performance. For all heavy load applications except vips, FULLY+WA performs best. Across these applications, FULLY+WA achieves an average of $21.3 \%$ and maximum $37.8 \%$ speedup over FULLY. With sufficient flexibility, FULLY+WA has an average $12.1 \%$ speedup over PSF+WA. The average speedups of FULLY+WA are $29.3 \%, 15.0 \%, 10.1 \%$, 


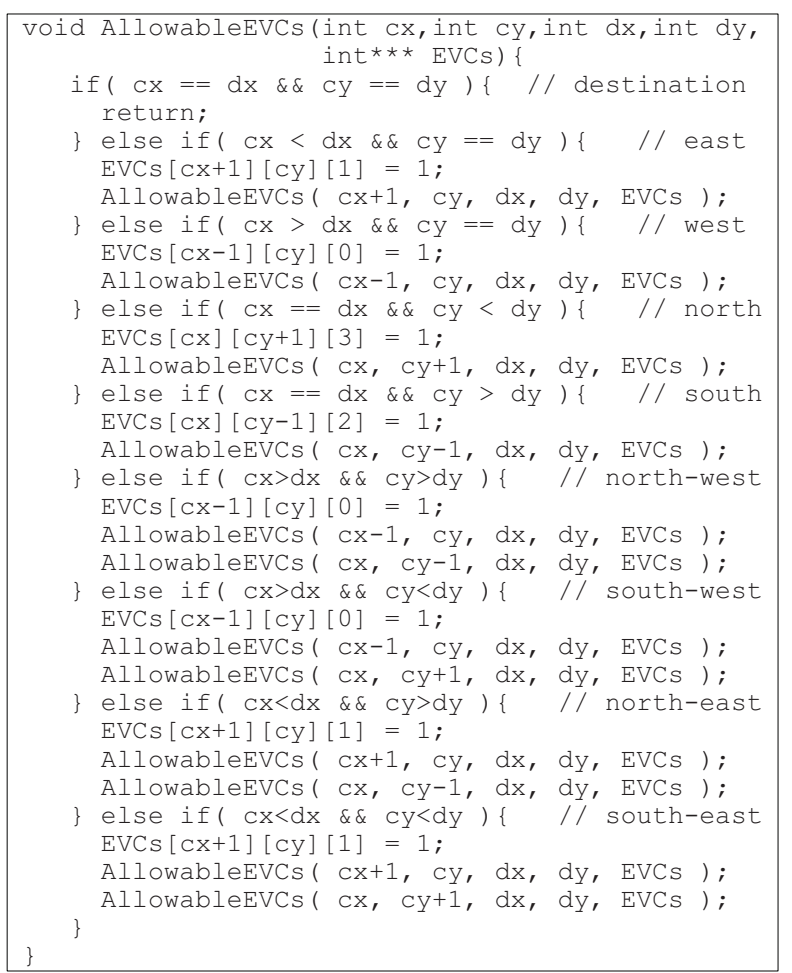

Fig. 4. The AllowableEVCs algorithm. The initial value of EVCs is 0s. cx and cy are $X$ and $Y$ positions of the current node. $d x$ and $d y$ are $X$ and $Y$ positions of the destination. Port encoding: East: 0, West: 1, South: 2, North: 3.

9.9\% and $10.4 \%$ over PSF, DOR, west-first, negativefirst and odd-even, respectively.

\section{Allowable Escape VCs (EVCs)}

In Sec. 5.2 of the main file, we leverage a recursive algorithm to calculate the allowable escape VCs (EVCs) for synthetic traffic patterns. Here, we present the algorithm, as shown in Fig. 4.

This algorithm recursively calculates all allowable EVCs for a packet sent from node $(c x, c y)$ to node $(d x, d y)$. At each step, the algorithm marks the escape VC at the port which adheres to DOR as allowable by setting the corresponding element of $E V C s$ array to ' 1 '. Fully adaptive routing may forward the packet to all neighbors inside the minimum quadrant defined by the current position and the destination. At the next step, the algorithm continues by using these neighbors as current positions. For example, if $c x>d x$ and $c y>d y$, then the destination is at the north-west quadrant of current position. The EVC at the east input port of node $(c x-1, c y)$ is allowed to be used. The packet may move to node $(c x-1, c y)$ and node $(c x, c y-1)$, and the algorithm continues by using them as current positions at the next step. By considering all source and destination pairs defined in the traffic pattern, we get the allowable EVCs in a $4 \times 4$ mesh network, as shown in TABLE 4 of the main file.

\section{Sensitivity to Network Design}

This section performs sensitivity study for network configuration parameters. Except for the analyzed
TABLE 2. Baseline configuration and variations.

\begin{tabular}{|l|l|l|}
\hline Characteristic & Baseline & Variations \\
\hline Topology (mesh) & $4 \times 4$ & $8 \times 8$ \\
\hline VCs/VN & 2 & 4 \\
\hline Flit buffers/VC & 4 & 3,2 \\
\hline SFP ratio & $80 \%$ & $60 \%, 40 \%$ \\
\hline
\end{tabular}

parameter, other parameters are the same as the baseline configuration shown in TABLE 2. This section makes comparison with the performance of baseline configuration shown in the main file. To improve the readability, we reproduce Fig. 10 of the main file, as shown in Fig. 5 here.

\subsection{Single-flit Packet Ratio (SFP ratio)}

Single-flit packet (SFP) ratios depend on the cache hierarchy, the coherence protocol and the application. To test the robustness of our design, we evaluate $60 \%$ and $40 \%$ SFP ratios for transpose- 1 . As shown in Fig. 6, DOR, west-first, negative-first and odd-even exhibit nearly identical performance for different SFP ratios. The aggressive VC re-allocation makes their performance insensitive to the packet length distribution. However, the performance of PSF and FULLY improves as the SFP ratio shrinks. Their conservative VC re-allocation favors long packets which utilize buffers more efficiently than short ones. As the SFP ratio decreases, so does the possibility of applying WPF. Thus, the performance gap between FULLY+WA and FULLY (or PSF+WA and PSF) decreases. However, even with a $40 \%$ SFP ratio, FULLY+WA achieves a $53.1 \%$ saturation throughput gain over FULLY.

\subsection{VC Depth}

Different NoCs may use different VC depths. To test the flexibility, we evaluate 3- and 2-flit deep VCs with bit reverse traffic. Comparing 4 flits/VC (Fig. 5a) and 3 flits/VC (Fig. 7a), DOR and west-first perform similarly, while FULLY and PSF show minor performance degradation. DOR and west-first offer no or very limited adaptiveness which is a major factor in their performance. Thus, reducing the VC depth from 4 to 3 has little effect. The bottleneck of FULLY and PSF is conservative VC re-allocation. Considering the majority of short packets, reducing the VC depth from 4 to 3 only affects performance slightly. However, the performance of FULLY+WA, PSF+WA, odd-even and negative-first declines with shallower VCs since the VC depth is their bottleneck. Shallow VCs increase the number of hops that a blocked packet spans, which increases the effect of chained blocking [23].

Comparing 3 and 2 flits/VC, performance drops for all algorithms. FULLY outperforms DOR and westfirst with 2 flits/VC. As the VC depth decreases, the difference between aggressive and conservative VC re-allocation declines; FULLY gets a relative gain. Even with 2 flits/VC, WPF still optimizes the performance since short packets dominate traffic. In Fig. 7b, FULLY+WA performs $46.2 \%$ better than FULLY. Novel flow control leads to superior performance even with 


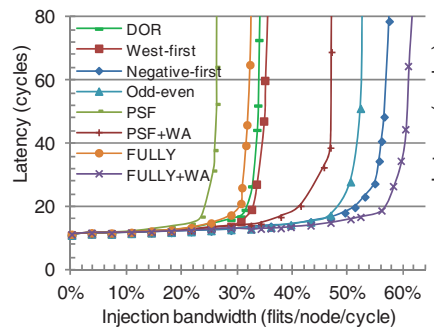

(a) Bit reverse.

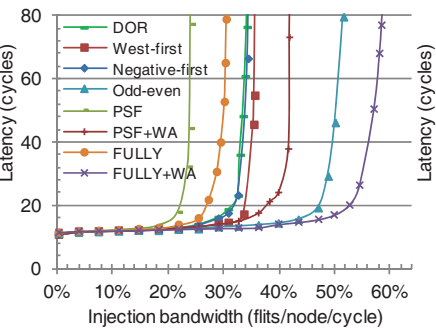

(b) Transpose-1.

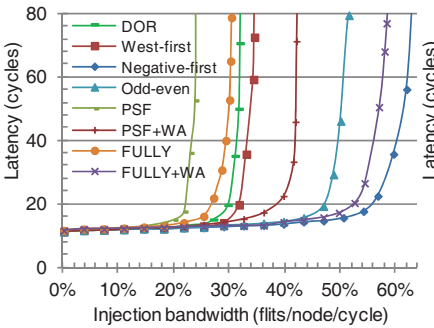

(c) Transpose-2.

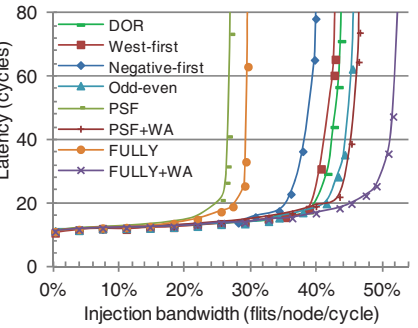

(d) Hotspot.

Fig. 5. Routing algorithm performance for the baseline configuration (A reproduction of Fig. 10 in the main file).

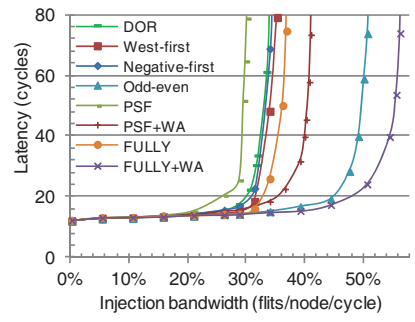

(a) $60 \%$ SFP ratio

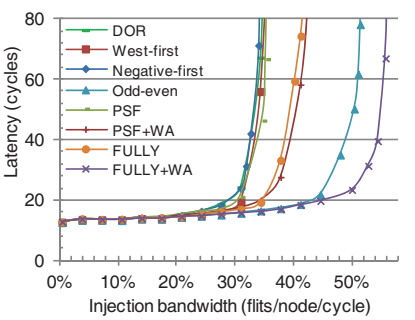

(b) $40 \%$ SFP ratio.

Fig. 6. The performance with different SFP ratios for transpose-1 traffic.

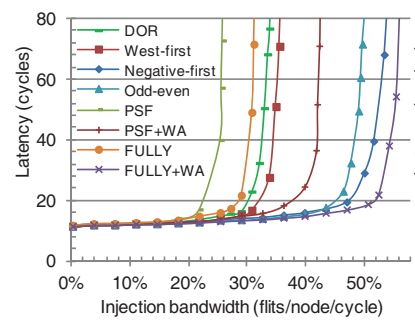

(a) 3-flit deep VCs.

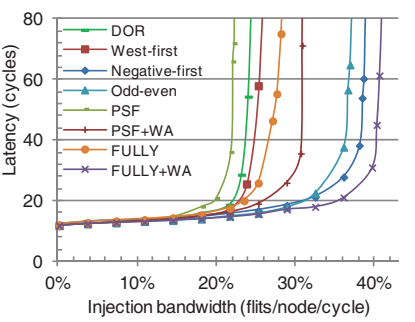

(b) 2-flit deep VCs.

Fig. 7. The performance with different VC depths for bit reverse traffic.

half of the buffers, which enables the design of a low-cost NoC. With 2 flits/VC (Fig. 7b), FULLY+WA's saturation throughput is $40.3 \%$, while FULLY's saturation throughput is $32.3 \%$ with 4 flits/VC (Fig. 5a). The same is true for PSF+WA.

\subsection{VC Count}

Semiconductor scaling and coherence protocol optimization may allow a VN to be configured with more VCs. Comparing 4 VCs/VN (Fig. 8a) and 2 VCs/VN (Fig. 5a), the performance of DOR, west-first and odd-even is almost the same. These algorithms offer limited adaptiveness; although additional results show increasing the VC count from 1 to 2 improves performance, increasing the VC count from 2 to 4 cannot reduce the physical path congestion and does not further improve performance. Negative-first has a modest gain. In contrast, PSF, FULLY, PSF+WA and FULLY+WA all have significant gains; more VCs mitigate the negative effects of conservative VC reallocation. The gap between PSF and FULLY (or PS$\mathrm{F}+\mathrm{WA}$ and FULLY+WA) decreases with more VCs; more VCs reduce the possibility of using escape VCs in PSF which forces packets to lose adaptivity.

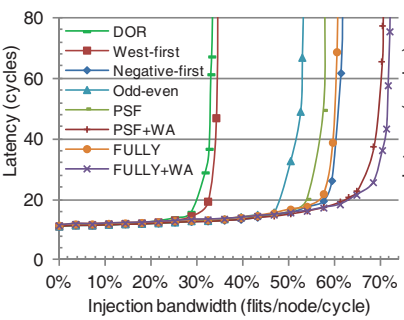

(a) Bit reverse.

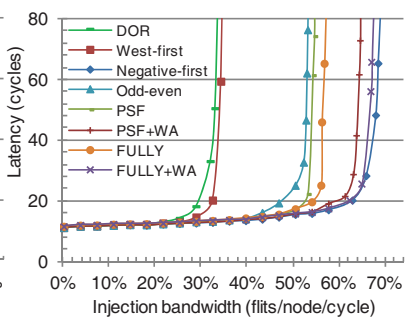

(b) Transpose-2.
Fig. 8. The performance with $4 \mathrm{VCs} / \mathrm{VN}$.

Fig. $8 \mathrm{~b}$ shows the performance of transpose-2, which is a favorable pattern for negative-first. FULLY+WA performs similarly to negative-first; with more VCs, the effect of restricting the use of escape VCs in FULLY+WA declines. More VCs reduces the gap between FULLY and FULLY+WA (or PSF and PSF+WA), since the possibility of facing empty VCs increases. Furthermore, using WPF to forward entire packets into non-empty VCs may result in Headof-Line (HoL) blocking [4] and limit FULLY+WA (or PSF+WA). Nevertheless, FULLY+WA still shows an average $19.8 \%$ gain over FULLY for these two patterns with 4 VCs; providing high VC utilization outweighs the negative effect of HoL blocking in a VC-limited NoC. Similar to VC depth, with only 2 VCs, FULLY+WA performs similarly or even better (Figs 5a and 5b) than FULLY with 4 VCs (Fig. 8). WPF provides similar or higher performance with half as many VCs.

\subsection{Network Size}

Fig. 9 explores the scalability for an $8 \times 8$ mesh. The trends across different algorithms are similar to the $4 \times 4$ mesh (Fig. 5). Communication is mostly determined by the traffic pattern. Since a larger network leads to higher average hop counts [14], it puts more pressure on VCs than a smaller one. Novel flow control achieves more gains in a larger network. The average improvement for these two patterns of FULLY+WA over FULLY is $108.2 \%$, while it is $93.1 \%$ in a $4 \times 4$ mesh. As packets travel more hops in a larger network, the possibility of entering an escape VC increases. For PSF and PSF+WA, once the packet enters an escape $\mathrm{VC}$, it loses adaptivity in subsequent hops. Therefore, the gap between FULLY+WA and PSF+WA (or FULLY and PSF) increases with a larger 


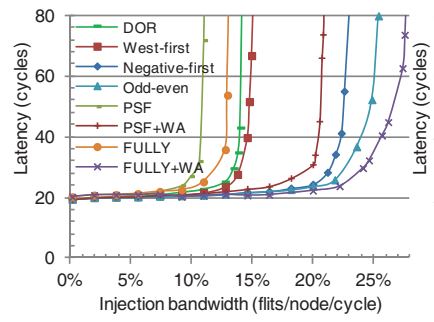

(a) Bit reverse.

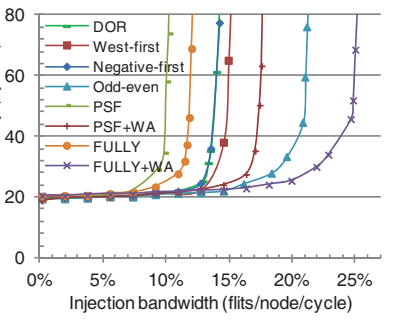

(b) Transpose-1.
Fig. 9. The performance for an $8 \times 8$ mesh network.

network; providing routing flexibility becomes more important with a larger network.

\section{FURTher Discussion}

\subsection{Packet Length}

Packet lengths for cache coherence traffic typically have a bimodal distribution. However, optimizations such as cache line compression [5], [11] create packet distributions that are not bimodal; the packet length may be distributed between a single flit and the maximum flits per packet supported by the architecture. To apply WPF on such NoCs, more downstream VC status registers are needed for the first-stage arbiters shown in Fig. 9 of the main file. An important consideration is how many different packet lengths to apply WPF to. The longest packet length that can use WPF is one flit shorter than the VC depth. Designers can ignore long packets, since there are few opportunities to apply WPF on long packets. This tradeoff depends on the packet length distribution, VC depth, hardware overhead and the expected performance gain.

\subsection{DAMQ and Hybrid Flow Controls}

Previous research proposed dynamically allocated multi-queue (DAMQ) designs for both off-chip [22] and on-chip networks [17], [26] to improve VC utilization. Even with DAMQ, allowing multiple packets to reside in one VC may lead to deadlock similar to Fig. 3 of the main file for fully adaptive routing in wormhole networks. WPF is complimentary to DAMQ as it ensures deadlock-freedom. WPF can be viewed as a hybrid mechanism combining wormhole and VCT. There are some hybrid flow controls [13], [19], [20]. Hybrid switching [19] and buffered wormhole [20] remove a blocked packet to release held physical channels by using either the processing node memory [19] or a central buffer [20]. Layered switching divides a long packet into several groups and tries to keep SA grants for a whole group [13]. Our research is different; we focus on improving the performance while avoiding deadlock.

\section{ACKNOWLEDGMENTS}

We thank the anonymous reviewers for their thorough and constructive suggestions. This work is supported by 863 Program of China (2012AA010905), NSFC (61272144, 61070037, 61272143, 61025009), the University of Toronto, NSERC of Canada, Research Project of NUDT (JC13-06-02).

\section{References}

[1] C. Bienia et al. The PARSEC benchmark suite: characterization and architectural implications. In PACT 2008.

[2] G. Chiu. The odd-even turn model for adaptive routing. IEEE Trans. Parallel Distrib. Syst., 11(7):729 -738, Jul. 2000.

[3] W. Dally and C. Seitz. Deadlock-free message routing in multiprocessor interconnection networks. IEEE Trans. Computers, C-36(5):547 -553, May 1987.

[4] W. Dally and B. Towles. Principles and Practices of Interconnection Networks. Morgan Kaufmann Publishers Inc., San Francisco, CA, USA, 2003.

[5] R. Das et al. Performance and power optimization through data compression in NoC architectures. In HPCA 2008.

[6] J. Duato. A new theory of deadlock-free adaptive routing in wormhole networks. IEEE Trans. Parallel Distrib. Syst., 4(12):1320 -1331, Dec. 1993.

[7] J. Duato. A necessary and sufficient condition for deadlockfree adaptive routing in wormhole networks. IEEE Trans. Parallel Distrib. Syst., 6(10):1055 -1067, Oct. 1995.

[8] E. Fleury and P. Fraigniaud. A general theory for deadlock avoidance in wormhole-routed networks. IEEE Trans. Parallel Distrib. Syst., 9:626-638, Jul. 1998.

[9] B. Fu, Y. Han, J. Ma, H. Li, and X. Li. An abacus turn model for time/space-efficient reconfigurable routing. In ISCA 2011.

[10] C. Glass and L. Ni. The turn model for adaptive routing. In ISCA 1992

[11] Y. Jin et al. Adaptive data compression for high-performance low-power on-chip networks. In MICRO 2008.

[12] X. Lin, P. McKinley, and L. Ni. The message flow model for routing in wormhole-routed networks. IEEE Trans. Parallel Distrib. Syst., 6(7):755 -760, Jul. 1995.

[13] Z. Lu, M. Liu, and A. Jantsch. Layered switching for networks on chip. In DAC 2007.

[14] S. Ma, N. Enright Jerger, and Z. Wang. DBAR: an efficient routing algorithm to support multiple concurrent applications in networks-on-chip. In ISCA 2011.

[15] P. Magnusson et al. Simics: A full system simulation platform. Computer, 35:50-58, Feb. 2002.

[16] N. Neelakantam et al. FeS2: A full-system execution-driven simulator for x86. In Poster presented at ASPLOS 2008, 2008.

[17] C. Nicopoulos et al. ViChaR: A dynamic virtual channel regulator for network-on-chip routers. In MICRO 2006.

[18] L. Schwiebert and D. N. Jayasimha. A necessary and sufficient condition for deadlock-free wormhole routing. J. Parallel Distrib. Comput., 32:103-117, Jan. 1996.

[19] K. Shin and S. Daniel. Analysis and implementation of hybrid switching. In ISCA 1995.

[20] C. Stunkel et al. The SP2 high-performance switch. IBM Syst. J., 34:185-204, Apr. 1995.

[21] S. Taktak, E. Encrenaz, and J. Desbarbieux. A polynomial algorithm to prove deadlock-freeness of wormhole networks. In PDP 2010.

[22] Y. Tamir and G. Frazier. High-performance multiqueue buffers for vlsi communication switches. In ISCA 1988.

[23] A. Vaidya, A. Sivasubramaniam, and C. Das. Impact of virtual channels and adaptive routing on application performance. IEEE Trans. Parallel Distrib. Syst., 12(2):223 -237, Feb. 2001.

[24] F. Verbeek and J. Schmaltz. Automatic verification for deadlock in networks-on-chips with adaptive routing and wormhole switching. In NoCS 2011.

[25] F. Verbeek and J. Schmaltz. On necessary and sufficient conditions for deadlock-free routing in wormhole networks. IEEE Trans. Parallel Distrib. Syst., 22(12):2022 -2032, Dec. 2011.

[26] Y. Xu et al. Simple virtual channel allocation for high throughput and high frequency on-chip routers. In HPCA 2010. 